Sociedad de Estadística e Investigación Operativa

Test (2000) Vol. 9, No. 2, pp. 439453

\title{
Mixture results for extremal behaviour of strongly dependent nonstationary Gaussian sequences
}

\author{
M. Graça Temido* \\ Department of Mathematics, University of Coimbra, and \\ C.E.A., University of Lisbon, Portugal.
}

\begin{abstract}
Let $\left\{X_{n}\right\}$ be a nonstationary Gaussian scquence. In this work we introduce a condition on $r_{i j}=\operatorname{Cor}\left(X_{i}, X_{j}\right), i, j \geq 1$ that models a strong dependence struture. We prove that the limit of the point process of excedances is a Cox process i.c. a point process whose distribution is a mixture of distributions of simple Poisson processes, regulated by a standard normal law. Moreover, we study the joint limit distribution of the maxima and minima, under linear normalization, and we again find a doubly stochastic behaviour.
\end{abstract}

Key Words: Cox process, exceedances, extremes, Gaussian processes, point processes.

AMS subject classification: $60 \mathrm{G} 70,60 \mathrm{G} 15$

\section{Introduction}

Extreme value theory for Gaussian sequences has been drawing the atention of many authors, for instance, Berman (1964), Mittal and Ylvisaker (1975), Rootzén (1983), Hüsler (1983), Leadbetter et al. (1983), who have been dealing with the limit distribution of suitable normalized extreme values.

Let $\left\{X_{n}\right\}$ be a nonstationary Gaussian sequence with $E\left(X_{j}\right)=m_{j}$, $\operatorname{Var}\left(X_{j}\right)=\sigma_{j}^{2}$ and correlations $r_{i j}, i, j \geq 1, i \neq j$. Define the maxima $M_{n}=\max _{1 \leq j \leq n} X_{j}$ and the minima $W_{n}=\min _{1 \leq j \leq n} X_{j}$.

It is well known that if $\left\{X_{n}\right\}$ is i.i.d. with standard marginal distribution, the maxima, with linear normalization, converges in distribution to a

\footnotetext{
"Correspondence to: Maria da Graca Temido, Departamento de Matemática, Faculdade de Ciencias e Tecnologia, Lniversidade de Coimbra. Apartado 3008 . 3000 Coimbra, Portugal. Email: mgtmōmat.uc.pt
}

This work was partially supported by JNICT/PRAXIS XXI/FEDER

Received: April 1999; Accepted: June 2000 